\title{
Periodic boundary value problems for fractional semilinear integro-differential equations with non-instantaneous impulses
}

\section{Bo Zhu ${ }^{1 *}$ and Lishan Liu',3}

\begin{abstract}
In this paper, we study periodic boundary value problems of fractional semilinear integro-differential equations with non-instantaneous impulses in Banach spaces. By the measure of noncompactness, the theory of $\beta$-resolvent family, and the fixed point theorem, we obtain several sufficient conditions on the existence of mild solutions for such problems. Finally, an example is given to show the main results of this paper.
\end{abstract}

MSC: 34A37; 34G20; 34K30; 35K90

Keywords: Fractional semilinear integro-differential equation; Measure of noncompactness; Non-instantaneous impulses; $\beta$-resolvent operator family

\section{Introduction}

In the last decades, many researchers have been attracted to studying the fractional differential equations, and a lot of good results have been obtained, see [1-17] and the references therein. In [1-9], authors studied the fractional differential equations with instantaneous impulses, which have been applied to describe abrupt changes such as the shocks and natural disasters. For more details on this subject, see [1-9]. The differential equations with instantaneous impulse cannot explain some dynamics problems of evolution process. For instance, the drug delivery in the bloodstream is a gradual and continuous process. However, the models with non-instantaneous impulses can explain these problems.

The differential equations with non-instantaneous impulses of the following form were initially investigated by the authors in $[18,19]$ :

$$
\left\{\begin{array}{l}
x^{\prime}(t)=A x(t)+f(t, x(t)), \quad t \in\left(s_{k}, t_{k+1}\right], k=0,1, \ldots, N, \\
x(t)=g_{k}(t, x(t)), \quad t \in\left(t_{k}, s_{k}\right], k=1,2, \ldots, N, \\
x(0)=x_{0} \in E,
\end{array}\right.
$$

where $A: D(A) \subset E \rightarrow E$ is the generator of a $C_{0}$-semigroup $\left\{S(t)_{t \geq 0}\right\}$ on a Banach space $E$. In $[18,19]$, the existence results have been established by using the fixed point theorems. 
In [20], authors investigated the following periodic boundary value problem of integer nonlinear evolution equations with non-instantaneous impulses:

$$
\begin{cases}x^{\prime}(t)=A x(t)+f(t, x(t)), & t \in\left(s_{k}, t_{k+1}\right], k=0,1, \ldots, N, \\ x(t)=S\left(t-t_{k}\right) g_{k}(t, x(t)), & t \in\left(t_{k}, s_{k}\right], k=1,2, \ldots, N, \\ x(0)=x(T), & \end{cases}
$$

where the semigroup $\left\{S(t)_{t \geq 0}\right\}$ is compact and the linear operator $A$ is independent of $t$. The existence results were obtained by using the fixed point theorems. Now, many researchers are studying the fractional differential equations with non-instantaneous impulses, and a lot of good results have been obtained [21-27]. In [21, 23], the authors studied the stability of the fractional differential equations with non-instantaneous impulses. The existence results of the fractional differential equations with non-instantaneous impulses are discussed by authors in [22, 24-26].

Inspired by the above said work, we consider the following periodic boundary value problem for nonlinear fractional evolution equations with non-instantaneous impulses:

$$
\left\{\begin{array}{l}
{ }^{c} D_{t}^{\beta} u(t)=A(t) u(t)+f(t, u(t))+\int_{0}^{t} q(t-s) h(s, u(s)) d s, \\
\quad t \in\left(s_{i}, t_{i+1}\right], i=0,1, \ldots, m, \\
u(t)=U_{\beta}\left(t, t_{i}\right) g_{i}(t, u(t)), \quad t \in\left(t_{i}, s_{i}\right], i=1,2, \ldots, m, \\
u(0)=u(T),
\end{array}\right.
$$

where ${ }^{c} D_{t}^{\beta}$ is the Caputo's fractional derivative of order $\beta \in(0,1], A(t)$ is dependent on $t$ and a closed and linear unbounded operator with domain $D(A)$ defined on a Banach space $E$, the fixed points $s_{i}$ and $t_{i}$ satisfying $0=s_{0}<t_{1} \leq s_{1}<t_{2} \leq \cdots<t_{m} \leq s_{m}<t_{m+1}=T$ are pre-fixed numbers. $f, U_{\beta}, h$, and $g_{i}(i=1,2, \ldots, m)$ are to be specified later, $q:[0, T] \rightarrow X$ is continuous.

In [18-20], differential equations are all integer order, the linear operator $A$ is independent of $t$, and the semigroup is compact. In [24-26], the linear operator $A$ is independent of $t$. In this paper, we consider the existence of mild solutions for the fractional differential equations (1.1) under the conditions of the compact and noncompact semigroup; meanwhile, the linear operator $A(t)$ is dependent on $t$. Therefore, the results presented in this paper improve and generalize the main results in [18-20,24-26] by using a different method.

\section{Preliminaries}

Let $J=[0, T], C(J, E)=\{u: J \rightarrow E$ is continuous $\}, \operatorname{PC}(J, E)=\left\{u: J \rightarrow E: u \in C\left(\left(s_{i}, t_{i+1}\right], E\right)\right.$, and there exist $u\left(t_{i}^{-}\right)$and $u\left(t_{i}^{+}\right)$with $\left.u\left(t_{i}^{-}\right)=u\left(t_{i}\right), i=1,2, \ldots, m\right\}$ with the PC-norm $\|u\|_{\mathrm{PC}}=\sup \{\|u(t)\|: t \in J\}$.

Lemma 2.1 ([28]) Let E be a Banach space, $D \subset E$ be a bounded closed and convex set. Assume that $Q: D \rightarrow D$ is a strict set contraction mapping. Then $Q$ has at least one fixed point in D. 
Definition 2.1 $([29,30])$ The Riemann-Liouville fractional integral of $f$ of order $v>0$ is defined by

$$
J_{t}^{v} f(t)=\frac{1}{\Gamma(v)} \int_{0}^{t}(t-s)^{\nu-1} f(s) d s, \quad f(t) \in L^{1}(J ; E) .
$$

The integral operators $\left\{J_{t}^{v}\right\}_{v \geq 0}$ have the following semigroup property:

$$
J_{t}^{\nu} J_{t}^{\mu}=J_{t}^{v+\mu}, \quad v, \mu \geq 0
$$

Definition $2.2([29,30])$ The Caputo fractional derivative of order $n-1<v<n$ with the lower limits zero for a function $f \in C^{n}[0,+\infty)$ can be written as

$$
{ }^{c} D_{t}^{v} f(t)=\frac{1}{\Gamma(n-v)} \int_{0}^{t}(t-s)^{n-\nu-1} f^{(n)}(s) d s, \quad t>0, n \in \mathbb{N} .
$$

Lemma 2.2 ([31]) Let $E$ be a Banach space and $B \subset C[J, E]$ be equicontinuous and bounded, then $\overline{\mathrm{Co}} B \subset C[J, E]$ is also equicontinuous and bounded (where $\overline{\mathrm{Co}} B$ denotes the closed convex hull of $B$ ).

Definition 2.3 ([32]) Let $S$ be a bounded set of $E, \alpha(S)=\inf \{\delta>0: S$ can be expressed as the union of a finite number of sets such that the diameter of each set does not exceed $\delta$, i.e., $S=\bigcup_{i=1}^{m} S_{i}$ with $\left.\operatorname{diam}\left(S_{i}\right) \leq \delta, i=1,2, \ldots, m\right\}, \alpha(S)$ is called the Kuratowski measure of noncompactness of set $S$. Obviously, $0<\alpha(S)<\infty$.

Lemma 2.3 ([33]) Let E be a Banach space, and let $D \subset E$ be bounded, then there exists a countable set $D_{0} \subset D$ such that $\alpha(D) \leq 2 \alpha\left(D_{0}\right)$.

Lemma 2.4 ([31, 34]) Let E be a Banach space, and let $B \subset C[, E]$ be equicontinuous and bounded, then $\alpha(B(t))$ is continuous on J, and

$$
\alpha\left(\int_{J} B(s) d s\right) \leq \int_{J} \alpha(B(s)) d s, \quad \alpha(B)=\max _{t \in J} \alpha(B(t)) .
$$

Definition 2.4 ([5,35]) Let $A(t)$ be a closed and linear operator with domain $D(A)$ defined on a Banach space $E, \beta>0$ be a constant. Let $\rho[A(t)]$ be the resolvent set of $A(t)$, we call $A(t)$ the generator of a $\beta$-resolvent operator family if there exist $\omega \geq 0$ and a strongly continuous function $U_{\beta}: \mathbb{R}_{+}^{2} \rightarrow B(E)$ such that $\left\{\lambda^{\beta}: \operatorname{Re} \lambda>\omega\right\} \subset \rho(A)$ and

$$
\left(\lambda^{\beta} I-A(s)\right)^{-1} u=\int_{0}^{\infty} e^{-\lambda(t-s)} U_{\beta}(t, s) u d t, \quad \operatorname{Re}(\lambda)>\omega, u \in E .
$$

In this case, $U_{\beta}(t, s)$ is called the $\beta$-resolvent family generated by $A(t)$.

Remark $2.1 \mathrm{By}[5,36]$, the $\beta$-resolvent operator family $U_{\beta}(t, s)$ satisfies the following properties:

(1) $U_{\beta}(s, s)=I, U_{\beta}(t, s)=U_{\beta}(t, r) U_{\beta}(r, s)$ for $0 \leq s \leq r \leq t \leq a$.

(2) $(t, s) \rightarrow U_{\beta}(t, s)$ is strongly continuous for $0 \leq s \leq t \leq a$. 
Definition 2.5 A function $u \in \operatorname{PC}(J, E)$ is said to be a mild solution of problem (1.1) if $u$ satisfies the following equations:

$$
\begin{aligned}
u(t)= & U_{\beta}(t, 0)\left[U_{\beta}\left(T, t_{m}\right) g_{m}\left(s_{m}, u\left(s_{m}\right)\right)\right. \\
& \left.+\int_{s_{m}}^{T} U_{\beta}(t, s)\left(f(s, u(s))+\int_{0}^{s} q(s-\tau) h(\tau, u(\tau)) d \tau\right) d s\right] \\
& +\int_{0}^{t} U_{\beta}(t, s)\left(f(s, u(s))+\int_{0}^{s} q(s-\tau) h(\tau, u(\tau)) d \tau\right) d s, \quad t \in\left[0, t_{1}\right], \\
u(t)= & U_{\beta}\left(t, t_{i}\right) g_{i}(t, u(t)), \quad t \in\left(t_{i}, s_{i}\right], i=1,2, \ldots, m, \\
u(t)= & U_{\beta}\left(t, t_{i}\right) g_{i}\left(s_{i}, u\left(s_{i}\right)\right)+\int_{s_{i}}^{t} U_{\beta}(t, s)\left(f(s, u(s))+\int_{0}^{s} q(s-\tau) h(\tau, u(\tau)) d \tau\right) d s, \\
t \in & \left(s_{i}, t_{i+1}\right], i=1, \ldots, m .
\end{aligned}
$$

\section{$3 U_{\beta}(t, s)$ is noncompact}

We define an operator $F: \operatorname{PC}(J, E) \rightarrow \operatorname{PC}(J, E)$ by

$$
(F u)(t)=\left\{\begin{array}{l}
U_{\beta}(t, 0)\left[U_{\beta}\left(T, t_{m}\right) g_{m}\left(s_{m}, u\left(s_{m}\right)\right)\right. \\
\left.\quad+\int_{s_{m}}^{T} U_{\beta}(T, s)\left(f(s, u(s))+\int_{0}^{s} q(s-\tau) h(\tau, u(\tau)) d \tau\right) d s\right] \\
\quad+\int_{0}^{t} U_{\beta}(t, s)\left(f(s, u(s))+\int_{0}^{s} q(s-\tau) h(\tau, u(\tau)) d \tau\right) d s, \quad t \in\left[0, t_{1}\right] \\
U_{\beta}\left(t, t_{i}\right) g_{i}(t, u(t)), \quad t \in\left(t_{i}, s_{i}\right], i=1,2, \ldots, m, \\
U_{\beta}\left(t, t_{i}\right) g_{i}\left(s_{i}, u\left(s_{i}\right)\right)+\int_{s_{i}}^{t} U_{\beta}(t, s)(f(s, u(s)) \\
\left.\quad+\int_{0}^{s} q(s-\tau) h(\tau, u(\tau)) d \tau\right) d s, \quad t \in\left(s_{i}, t_{i+1}\right], i=1, \ldots, m .
\end{array}\right.
$$

Theorem 3.1 Assume that the following conditions $\left(H_{1}\right)$ and $\left(H_{2}\right)$ hold.

$\left(H_{1}\right)$ For any $R>0$, functions $f, h$ and $g_{i}(i=1,2, \ldots, m): J \times E \rightarrow E$ are bounded and continuous on $J \times T_{R}$, and

$$
\limsup _{R \rightarrow \infty} \frac{M(R)}{R}<\frac{1}{\Delta}
$$

where

$$
\begin{aligned}
\Delta= & \max \left\{M^{2}\left(1+\left(T-s_{m}\right)+\int_{s_{m}}^{T} \int_{0}^{s} q(s-\tau) d \tau d s\right)+M\left(t_{1}+\int_{0}^{t} \int_{0}^{s} q(s-\tau) d \tau d s\right),\right. \\
& \left.M, M\left(1+\left(t_{i+1}-s_{i}\right)+\int_{s_{i}}^{t} \int_{0}^{s} q(s-\tau) d \tau d s\right), i=1,2, \ldots, m\right\},
\end{aligned}
$$

$M(R)=\sup \left\{\|f(t, u)\|,\|h(t, u)\|,\left\|g_{i}(t, u)\right\|(i=1,2, \ldots, m):(t, u) \in J \times T_{R}\right\}, T_{R}=$ $\{u \in E:\|u\| \leq R\}$, the resolvent operator $U_{\beta}(t, s)$ is noncompact for $t, s>0, M=$ $\max _{0 \leq s<t \leq T}\left\|U_{\beta}(t, s)\right\|<+\infty$.

$\left(H_{2}\right)$ For all $R>0$, there exist nonnegative Lebesgue integrable functions $L_{f}, L_{h}, L_{g_{i}} \in$ $L^{1}\left(J, \mathbb{R}^{+}\right)(i=1,2, \ldots, m)$ such that, for all equicontinuous and countable sets $D \subset$ 


$$
\begin{aligned}
T_{R}= & \{u \in E:\|u\| \leq R\}, \\
& \alpha\left(f(t, D) \leq L_{f}(t) \alpha(D), \quad \alpha\left(h(t, D) \leq L_{h}(t) \alpha(D)\right.\right.
\end{aligned}
$$

and

$$
\alpha\left(g_{i}(t, D) \leq L_{g_{i}}(t) \alpha(D)\right.
$$

with

$$
\begin{aligned}
\varrho= & \max \left\{M^{2} L_{g_{m}}(t)+M^{2} \int_{s_{m}}^{T} L_{f}(s) d s+M \int_{0}^{t} L_{f}(s) d s\right. \\
& +\left(M^{2}+M\right) \int_{0}^{t} \int_{0}^{s} q(s-\tau) L_{h}(\tau) d \tau d s, \\
& M L_{g_{i}}(t), L_{g_{i}}(t)+\int_{s_{i}}^{t} L_{f}(s) d s+\int_{s_{i}}^{t} \int_{0}^{s} q(s-\tau) L_{h}(\tau) d \tau d s, \\
& i=1,2,3, \ldots, m\} \\
< & 1 .
\end{aligned}
$$

Then problem (1.1) has at least one mild solution $u \in \mathrm{PC}(J, E)$.

Proof By (3.2), there exist $0<r<\Delta^{-1}$ and $R_{0}>0$ such that, for any $R \geq R_{0}$, we have

$$
M(R)<r R .
$$

Let $R^{*} \geq R_{0}$. For any $u \in B_{R^{*}}=\left\{u \in \operatorname{PC}(J, E):\|u\|_{\mathrm{PC}} \leq R^{*}\right\}$, we prove that $F u \in B_{R^{*}}$.

Step 1. By (3.2), for any $t \in\left[0, t_{1}\right]$, we have

$$
\begin{aligned}
\|(F u)(t)\| \leq & \left\|U_{\beta}(t, 0)\right\|\left\|U_{\beta}\left(T, t_{m}\right) g_{m}\left(s_{m}, u\left(s_{m}\right)\right)\right\| \\
& +\left\|U_{\beta}(t, 0)\right\|\left\|\int_{s_{m}}^{T} U_{\beta}(T, s)\left(f(s, u(s))+\int_{0}^{s} q(s-\tau) h(\tau, u(\tau)) d \tau\right) d s\right\| \\
& +\left\|\int_{0}^{t} U_{\beta}(t, s)\left(f(s, u(s))+\int_{0}^{s} q(s-\tau) h(\tau, u(\tau)) d \tau\right) d s\right\| \\
\leq & M^{2} r R^{*}\left(1+\left(T-s_{m}\right)+\int_{s_{m}}^{T} \int_{0}^{s} q(s-\tau) d \tau d s\right) \\
& +M^{*} R^{*}\left(t_{1}+\int_{0}^{t} \int_{0}^{s} q(s-\tau) d \tau d s\right) \\
\leq & R^{*} .
\end{aligned}
$$

For any $t \in\left(t_{i}, s_{i}\right], i=1,2, \ldots, m$, we have

$$
\|(F u)(t)\| \leq\left\|U_{\beta}\left(t, t_{i}\right) g_{i}(t, u(t))\right\| \leq M r R^{*} \leq R^{*} .
$$


For any $t \in\left(s_{i}, t_{i+1}\right], i=1,2, \ldots, m$, we have

$$
\begin{aligned}
\|(F u)(t)\| \leq & \left\|U_{\beta}\left(t, t_{i}\right) g_{i}\left(s_{i}, u\left(s_{i}\right)\right)\right\| \\
& +\left\|\int_{s_{i}}^{t} U_{\beta}(t, s)\left(f(s, u(s))+\int_{0}^{s} q(s-\tau) h(\tau, u(\tau)) d \tau\right) d s\right\| \\
\leq & M r R^{*}\left(1+\left(t_{i+1}-s_{i}\right)+\int_{s_{i}}^{t} \int_{0}^{s} q(s-\tau) d \tau d s\right) \\
\leq & R^{*} .
\end{aligned}
$$

Step 2. We prove that $F: B_{R^{*}} \rightarrow B_{R^{*}}$ is continuous. Let $\left\{u_{n}\right\}_{0}^{\infty} \subset \mathrm{PC}(J, E)$ and $u_{n} \rightarrow u$, $u \in \mathrm{PC}(J, E)$. By the continuity of the functions $f, g_{i}(i=1,2, \ldots, m), h$, we have

$$
\begin{aligned}
& \lim _{n \rightarrow \infty} \sup _{t \in J}\left\|f\left(t, u_{n}(t)\right)-f(t, u(t))\right\|=0, \\
& \lim _{n \rightarrow \infty} \sup _{t \in J}\left\|g_{i}\left(t, u_{n}(t)\right)-g_{i}(t, u(t))\right\|=0, \\
& \lim _{n \rightarrow \infty} \sup _{t \in J}\left\|h\left(t, u_{n}(t)\right)-h(t, u(t))\right\|=0 .
\end{aligned}
$$

For any $t \in\left[0, t_{1}\right]$, we have

$$
\begin{aligned}
& \left\|\left(F u_{n}\right)(t)-(F u)(t)\right\| \\
& \leq M^{2} \sup _{t \in J}\left\|g_{m}\left(t, u_{n}(t)\right)-g_{m}(t, u(t))\right\| \\
& \quad+M^{2} \int_{s_{m}}^{T}\left\|f\left(s, u_{n}(s)\right)-f(s, u(s))\right\| d s \\
& \quad+M^{2} \int_{s_{m}}^{T} \int_{0}^{s}\left\|h\left(s, u_{n}(s)\right)-h(s, u(s))\right\| q(s-\tau) d \tau d s \\
& \quad+M t_{1} \sup _{t \in J}\left\|f\left(t, u_{n}(t)\right)-f(t, u(t))\right\| \\
& \quad+M \int_{0}^{T} \int_{0}^{s}\left\|h\left(s, u_{n}(s)\right)-h(s, u(s))\right\| q(s-\tau) d \tau d s .
\end{aligned}
$$

For any $t \in\left(t_{i}, s_{i}\right], i=1,2, \ldots, m$, we have

$$
\left\|\left(F u_{n}\right)(t)-(F u)(t)\right\| \leq M \sup _{t \in J}\left\|g_{i}\left(t, u_{n}(t)\right)-g_{i}(t, u(t))\right\| .
$$

For any $t \in\left(s_{i}, t_{i+1}\right], i=1,2, \ldots, m$, we have

$$
\begin{aligned}
\left\|\left(F u_{n}\right)(t)-(F u)(t)\right\| \leq & M \sup _{t \in J}\left\|g_{i}\left(t, u_{n}(t)\right)-g_{i}(t, u(t))\right\| \\
& +M \int_{s_{i}}^{T}\left\|f\left(s, u_{n}(s)\right)-f(s, u(s))\right\| d s \\
& +M \int_{s_{i}}^{T} \int_{0}^{s}\left\|h\left(s, u_{n}(s)\right)-h(s, u(s))\right\| q(s-\tau) d \tau d s .
\end{aligned}
$$


By the above inequalities, we know that

$$
\left\|F u_{n}-F u\right\|_{\mathrm{PC}} \rightarrow 0 \quad(n \rightarrow \infty)
$$

Therefore, the operator $F: B_{R^{*}} \rightarrow B_{R^{*}}$ is continuous.

Step 3. We will prove that $F\left(B_{R^{*}}\right)$ is equicontinuous. Case 1 . For interval $\left[0, t_{1}\right], 0 \leq e_{1}<$ $e_{2} \leq t_{1}, u \in B_{R^{*}}$, we have

$$
\begin{aligned}
\left\|(F u)\left(e_{2}\right)-(F u)\left(e_{1}\right)\right\| \\
\leq\left\|U_{\beta}\left(e_{2}, 0\right)-U_{\beta}\left(e_{1}, 0\right)\right\|\left\|U_{\beta}\left(T, t_{m}\right) g_{m}\left(s_{m}, u\left(s_{m}\right)\right)\right\| \\
+\left\|U_{\beta}\left(e_{2}, 0\right)-U_{\beta}\left(e_{1}, 0\right)\right\| \\
\quad \times\left\|\int_{s_{m}}^{T} U_{\beta}(T, s)\left(f(s, u(s))+\int_{0}^{s} q(s-\tau) h(\tau, u(\tau)) d \tau\right) d s\right\| \\
+\sup _{s \in\left[0, t_{1}\right]}\left\|U_{\beta}\left(e_{2}, s\right)-U_{\beta}\left(e_{1}, s\right)\right\| \\
\quad \times\left\|\int_{0}^{e_{1}}\left(f(s, u(s))+\int_{0}^{s} q(s-\tau) h(\tau, u(\tau)) d \tau\right) d s\right\| \\
+\left\|\int_{e_{1}}^{e_{2}} U_{\beta}\left(e_{2}, s\right)\left(f(s, u(s))+\int_{0}^{s} q(s-\tau) h(\tau, u(\tau)) d \tau\right) d s\right\| .
\end{aligned}
$$

Case 2. For interval $\left(t_{i}, s_{i}\right], i=1,2, \ldots, m, 0 \leq e_{1}<e_{2} \leq t_{1}, u \in B_{R^{*}}$, we have

$$
\begin{aligned}
& \left\|(F u)\left(e_{2}\right)-(F u)\left(e_{1}\right)\right\| \\
& \quad \leq\left\|U_{\beta}\left(e_{2}, t_{i}\right) g_{i}\left(e_{2}, u\left(e_{2}\right)\right)-U_{\beta}\left(e_{1}, t_{i}\right) g_{i}\left(e_{1}, u\left(e_{1}\right)\right)\right\| \\
& \quad \leq\left\|U_{\beta}\left(e_{2}, e_{1}\right) U_{\beta}\left(e_{1}, t_{i}\right) g_{i}\left(e_{2}, u\left(e_{2}\right)\right)-U_{\beta}\left(e_{1}, t_{i}\right) g_{i}\left(e_{1}, u\left(e_{1}\right)\right)\right\| \\
& \quad \leq M\left\|U_{\beta}\left(e_{2}, e_{1}\right) g_{i}\left(e_{2}, u\left(e_{2}\right)\right)-g_{i}\left(e_{1}, u\left(e_{1}\right)\right)\right\| .
\end{aligned}
$$

Case 3. For interval $\left(s_{i}, t_{i+1}\right], i=1,2, \ldots, m, 0 \leq e_{1}<e_{2} \leq t_{1}, u \in B_{R^{*}}$, we have

$$
\begin{aligned}
& \left\|(F u)\left(e_{2}\right)-(F u)\left(e_{1}\right)\right\| \\
& \leq\left\|U_{\beta}\left(e_{2}, t_{i}\right)-U_{\beta}\left(e_{1}, t_{i}\right)\right\|\left\|g_{i}\left(s_{i}, u\left(s_{i}\right)\right)\right\| \\
& \quad+\sup _{s \in\left(s_{i}, t_{i+1}\right]}\left\|U_{\beta}\left(e_{2}, s\right)-U_{\beta}\left(e_{1}, s\right)\right\| \\
& \quad \times \int_{s_{i}}^{e_{1}}\left(f(s, u(s))+\int_{0}^{s} q(s-\tau) h(\tau, u(\tau)) d \tau\right) d s \\
& +\left\|\int_{e_{1}}^{e_{2}} U_{\beta}\left(e_{2}, s\right)\left(f(s, u(s))+\int_{0}^{s} q(s-\tau) h(\tau, u(\tau)) d \tau\right) d s\right\| .
\end{aligned}
$$

By (1)-(2) of Remark 2.1 and the above inequalities (3.6)-(3.8), we derive that $\|(F u)\left(e_{2}\right)-$ $(F u)\left(e_{1}\right) \| \rightarrow 0$ as $e_{2} \rightarrow e_{1}$. Therefore $F\left(B_{R^{*}}\right)$ is equicontinuous. From Lemma 2.2, we know that $\overline{\operatorname{Co}} F\left(B_{R^{*}}\right) \subset B_{R^{*}}$ is bounded and equicontinuous. 
Step 4. We will prove that $F: \overline{\mathrm{Co}} F\left(B_{R^{*}}\right) \rightarrow \overline{\mathrm{Co}} F\left(B_{R^{*}}\right)$ is a condensing operator. For any $D \subset \overline{\mathrm{Co}} F\left(B_{R^{*}}\right)$, by Lemma 2.3 , there exists a countable set $D_{0}=\left\{u_{n}\right\} \subset D$ such that

$$
\alpha(F(D)) \leq 2 \alpha\left(F\left(D_{0}\right)\right)
$$

From the equicontinuity of $\overline{\mathrm{Co}} F\left(B_{R^{*}}\right)$, we have that $D_{0} \subset \overline{\mathrm{Co}} F\left(B_{R^{*}}\right)$ is equicontinuous. Case 1 . For any $t \in\left[0, t_{1}\right]$, by Lemma 2.4 , we get

$$
\begin{aligned}
\alpha\left(F\left(D_{0}\right)(t)\right) \leq & M^{2} \alpha\left(g_{m}\left(s_{m}, D_{0}\left(s_{m}\right)\right)\right) \\
& +M^{2} \alpha\left(\int_{s_{m}}^{T}\left(f\left(s, D_{0}(s)\right)+\int_{0}^{s} q(s-\tau) h\left(\tau, D_{0}(\tau)\right) d \tau\right) d s\right) \\
& +M \alpha\left(\int_{0}^{t}\left(f\left(s, D_{0}(s)\right)+\int_{0}^{s} q(s-\tau) h\left(\tau, D_{0}(\tau)\right) d \tau\right) d s\right) \\
\leq & M^{2} L_{g_{m}}(t) \alpha(D) \\
& +M^{2}\left(\int_{s_{m}}^{T} L_{f}(s) \alpha\left(D_{0}(s)\right)+\int_{0}^{s} q(s-\tau) L_{h}(\tau) \alpha\left(D_{0}(\tau)\right) d \tau d s\right) \\
& +M\left(\int_{0}^{t} L_{f}(s) \alpha\left(D_{0}(s)\right)+\int_{0}^{s} q(s-\tau) L_{h}(\tau) \alpha\left(D_{0}(\tau)\right) d \tau d s\right) \\
\leq & \left(M^{2} L_{g_{m}}(t)+M^{2} \int_{s_{m}}^{T} L_{f}(s) d s+M \int_{0}^{t} L_{f}(s) d s\right. \\
+ & \left.\left(M^{2}+M\right) \int_{0}^{t} \int_{0}^{s} q(s-\tau) L_{h}(\tau) d \tau d s\right) \alpha(D) .
\end{aligned}
$$

Case 2. For any $t \in\left(t_{i}, s_{i}\right], i=1,2, \ldots, m$, we get

$$
\alpha\left(F\left(D_{0}\right)(t)\right) \leq M L_{g_{i}}(t) \alpha(D) .
$$

Case 3. For any $t \in\left(s_{i}, t_{i+1}\right], i=1,2, \ldots, m$, we get

$$
\begin{aligned}
\alpha\left(F\left(D_{0}\right)(t)\right) \leq & M L_{g_{i}}(t) \alpha(D) \\
& +M\left(\int_{s_{i}}^{t} L_{f}(s) \alpha\left(D_{0}(s)\right)+\int_{0}^{s} q(s-\tau) L_{h}(\tau) \alpha\left(D_{0}(\tau)\right) d \tau d s\right) \\
\leq & M\left(L_{g_{i}}(t)+\int_{s_{i}}^{t} L_{f}(s) d s+\int_{s_{i}}^{t} \int_{0}^{s} q(s-\tau) L_{h}(\tau) d \tau d s\right) \alpha(D) .
\end{aligned}
$$

By Lemma 2.4, we have

$$
\alpha\left(F\left(D_{0}\right)\right)=\max _{t \in J} \alpha\left(F\left(D_{0}\right)(t)\right) .
$$

Therefore

$$
\alpha(F(D)) \leq \varrho \alpha(D)
$$


By (3.5), we get that $F: \overline{\mathrm{Co}} F\left(B_{R^{*}}\right) \rightarrow \overline{\mathrm{Co}} F\left(B_{R^{*}}\right)$ is a strict set contraction mapping. Thus, by Lemma 2.1, $F$ has at least one fixed point $u^{*} \in \overline{\operatorname{Co}} F\left(B_{R^{*}}\right) \subset \operatorname{PC}(J, E)$, which means that problem (1.1) has at least one mild solution.

Remark 3.1 In Theorem 3.1, we assume that the resolvent operator $U_{\beta}(t, s)$ is noncompact for $t>0$. In the following Theorem 4.1, the resolvent operator $U_{\beta}(t, s)$ is compact for $t>0$.

\section{$4 U_{\beta}(t, s)$ is compact}

Theorem 4.1 Assume that the following conditions $\left(H_{3}\right)-\left(H_{5}\right)$ hold.

$\left(H_{3}\right)$ The resolvent operator $U_{\beta}(t, s)$ is compact for $t, s>0$ and $M=$ $\max _{0 \leq s<t \leq T}\left\|U_{\beta}(t, s)\right\|<+\infty$.

$\left(H_{4}\right)$ The functions $f, h: J \times E \rightarrow E$ are continuous, and there exist nonnegative Lebesgue integrable functions $a, b, L_{f}, L_{h} \in L^{1}\left(J, \mathbb{R}^{+}\right)$such that, for all $u \in E, t \in J$,

$$
\begin{array}{ll}
\|f(t, u)\| \leq a(t)+L_{f}(t)\|u\|^{\gamma}, & 0<\gamma \leq 1, \\
\|h(t, u)\| \leq b(t)+L_{h}(t)\|u\|^{\mu}, & 0<\mu \leq 1 .
\end{array}
$$

$\left(H_{5}\right)$ The functions $g_{i}, i=1,2, \ldots, m: J \times E \rightarrow E$ are continuous, there exist nonnegative functions $c_{i}, L_{g_{i}}, i=1,2, \ldots, m$, such that

$$
\left\|g_{i}(t, u)\right\| \leq c_{i}(t)+L_{g_{i}}(t)\|u\|^{v_{i}}, \quad 0<v_{i} \leq 1, i=1,2, \ldots, m,
$$

and

$$
\bar{C}=\sup _{t \in\left[t_{i}, s_{i}\right], i=1,2, \ldots, m} c_{i}(t)<\infty, \quad \bar{L}=\sup _{t \in\left[t_{i}, s_{i}\right], i=1,2, \ldots, m} L_{g_{i}}(t)<\infty .
$$

Then problem (1.1) has a mild solution on $\mathrm{PC}(J, E)$.

Proof The proof includes the following several steps.

Step 1. $F: \operatorname{PC}(J, E) \rightarrow \operatorname{PC}(J, E)$ is continuous. The proof is similar to Step 1 of Theorem 3.1.

Step 2. For all $R>0$, we will prove that $F\left(B_{R}\right)$ is equicontinuous. Case 1. For interval $\left[0, t_{1}\right], 0 \leq e_{1}<e_{2} \leq t_{1}, u \in B_{R}$, we get

$$
\begin{aligned}
&\left\|(F u)\left(e_{2}\right)-(F u)\left(e_{1}\right)\right\| \\
& \leq\left\|U_{\beta}\left(e_{2}, 0\right)-U_{\beta}\left(e_{1}, 0\right)\right\|\left\|U_{\beta}\left(T, t_{m}\right) g_{m}\left(s_{m}, u\left(s_{m}\right)\right)\right\| \\
&+\left\|U_{\beta}\left(e_{2}, 0\right)-U_{\beta}\left(e_{1}, 0\right)\right\| \\
& \times\left\|\int_{s_{m}}^{T} U_{\beta}(T, s)\left(f(s, u(s))+\int_{0}^{s} q(s-\tau) h(\tau, u(\tau)) d \tau\right) d s\right\| \\
&+\sup _{s \in\left[0, t_{1}\right]}\left\|U_{\beta}\left(e_{2}, s\right)-U_{\beta}\left(e_{1}, s\right)\right\| \\
& \times\left\|\int_{0}^{e_{1}}\left(f(s, u(s))+\int_{0}^{s} q(s-\tau) h(\tau, u(\tau)) d \tau\right) d s\right\| \\
&+\left\|\int_{e_{1}}^{e_{2}} U_{\beta}\left(e_{2}, s\right)\left(f(s, u(s))+\int_{0}^{s} q(s-\tau) h(\tau, u(\tau)) d \tau\right) d s\right\|
\end{aligned}
$$




$$
\begin{aligned}
\leq & M\left(\bar{C}+\bar{L} R^{v_{m}}\right)\left\|U_{\beta}\left(e_{2}, 0\right)-U_{\beta}\left(e_{1}, 0\right)\right\| \\
& +M\left\|U_{\beta}\left(e_{2}, 0\right)-U_{\beta}\left(e_{1}, 0\right)\right\| \\
& \times \int_{s_{m}}^{T}\left(a(s)+L_{f}(s) R^{\gamma}+\int_{0}^{s} q(s-\tau)\left(b(\tau)+L_{h}(\tau) R^{\mu}\right) d \tau\right) d s \\
& +\sup _{s \in\left[0, t_{1}\right]}\left\|U_{\beta}\left(e_{2}, s\right)-U_{\beta}\left(e_{1}, s\right)\right\| \\
& \times \int_{0}^{e_{1}}\left(a(s)+L_{f}(s) R^{\gamma}+\int_{0}^{s} q(s-\tau)\left(b(\tau)+L_{h}(\tau) R^{\mu}\right) d \tau\right) d s \\
& +M \int_{e_{1}}^{e_{2}}\left(a(s)+L_{f}(s) R^{\gamma}+\int_{0}^{s} q(s-\tau)\left(b(\tau)+L_{h}(\tau) R^{\mu}\right) d \tau\right) d s .
\end{aligned}
$$

Case 2. For interval $\left(t_{i}, s_{i}\right], i=1,2, \ldots, m, 0 \leq e_{1}<e_{2} \leq t_{1}, u \in B_{R}$, we have

$$
\begin{aligned}
\left\|(F u)\left(e_{2}\right)-(F u)\left(e_{1}\right)\right\| & \leq\left\|U_{\beta}\left(e_{2}, t_{i}\right) g_{i}\left(e_{2}, u\left(e_{2}\right)\right)-U_{\beta}\left(e_{1}, t_{i}\right) g_{i}\left(e_{1}, u\left(e_{1}\right)\right)\right\| \\
& \leq M\left\|U_{\beta}\left(e_{2}, e_{1}\right) g_{i}\left(e_{2}, u\left(e_{2}\right)\right)-g_{i}\left(e_{1}, u\left(e_{1}\right)\right)\right\| .
\end{aligned}
$$

Case 3. For interval $\left(s_{i}, t_{i+1}\right], i=1,2, \ldots, m, 0 \leq e_{1}<e_{2} \leq t_{1}, u \in B_{R}$, we have

$$
\begin{aligned}
& \left\|(F u)\left(e_{2}\right)-(F u)\left(e_{1}\right)\right\| \\
& \leq\left\|U_{\beta}\left(e_{2}, t_{i}\right)-U_{\beta}\left(e_{1}, t_{i}\right)\right\|\left\|g_{i}\left(s_{i}, u\left(s_{i}\right)\right)\right\|+\sup _{s \in\left(s_{i}, t_{i+1}\right]}\left\|U_{\beta}\left(e_{2}, s\right)-U_{\beta}\left(e_{1}, s\right)\right\| \\
& \quad \times\left\|\int_{s_{i}}^{e_{1}}\left(f(s, u(s))+\int_{0}^{s} q(s-\tau) h(\tau, u(\tau)) d \tau\right) d s\right\| \\
& \quad+\left\|\int_{e_{1}}^{e_{2}} U_{\beta}\left(e_{2}, s\right)\left(f(s, u(s))+\int_{0}^{s} q(s-\tau) h(\tau, u(\tau)) d \tau\right) d s\right\| \\
& \leq\left(\bar{C}+\bar{L} R^{v_{i}}\right)\left\|U_{\beta}\left(e_{2}, t_{i}\right)-U_{\beta}\left(e_{1}, t_{i}\right)\right\|+\sup _{s \in\left(s_{i}, t_{i+1}\right]}\left\|U_{\beta}\left(e_{2}, s\right)-U_{\beta}\left(e_{1}, s\right)\right\| \\
& \quad \times \int_{s_{i}}^{e_{1}}\left(a(s)+L_{f}(s) R^{\gamma}+\int_{0}^{s} q(s-\tau)\left(b(\tau)+L_{h}(\tau) R^{\mu}\right) d \tau\right) d s \\
& \quad+M \int_{e_{1}}^{e_{2}}\left(a(s)+L_{f}(s) R^{\gamma}+\int_{0}^{s} q(s-\tau)\left(b(\tau)+L_{h}(\tau) R^{\mu}\right) d \tau\right) d s .
\end{aligned}
$$

By Remark 2.1 and the above inequalities (4.1)-(4.3), we derive that $\left\|(F u)\left(e_{2}\right)-(F u)\left(e_{1}\right)\right\| \rightarrow$ 0 as $e_{2} \rightarrow e_{1}$. Therefore, $F\left(B_{R}\right)$ is equicontinuous.

Step 3. We will prove that $F\left(B_{R}\right)$ is precompact. For all fixed $t(0<t \leq T)$ and $0<\epsilon<t$, let $u \in B_{R}$ and define

$$
\left(F_{\epsilon} u\right)(t)=\left\{\begin{array}{l}
U_{\beta}(t, 0)\left[U_{\beta}\left(T, t_{m}\right) g_{m}\left(s_{m}, u\left(s_{m}\right)\right)\right. \\
\left.\quad+\int_{s_{m}}^{T} U_{\beta}(T, s)\left(f(s, u(s))+\int_{0}^{s} q(s-\tau) h(\tau, u(\tau)) d \tau\right) d s\right] \\
\quad+\int_{0}^{t-\epsilon} U_{\beta}(t, s)\left(f(s, u(s))+\int_{0}^{s} q(s-\tau) h(\tau, u(\tau)) d \tau\right) d s, \quad t \in\left[0, t_{1}\right], \\
U_{\beta}\left(t, t_{i}\right) g_{i}(t, u(t)), \quad t \in\left(t_{i}, s_{i}\right], i=1,2, \ldots, m, \\
U_{\beta}\left(t, t_{i}\right) g_{i}\left(s_{i}, u\left(s_{i}\right)\right)+\int_{s_{i}}^{t-\epsilon} U_{\beta}(t, s)\left(f(s, u(s))+\int_{0}^{s} q(s-\tau) h(\tau, u(\tau)) d \tau\right) d s \\
\quad t \in\left(s_{i}, t_{i+1}\right], i=1, \ldots, m .
\end{array}\right.
$$


By the compactness of $U_{\beta}(t, s)$ for $t, s>0$, the set $Y_{\epsilon}(t)=\left\{\left(F_{\epsilon} u\right)(t): u \in B_{R}\right\}$ is relatively compact in $E$ for any $\epsilon(0<\epsilon<t)$. For any $u \in B_{R}$, from the following inequalities

$$
\left\|(F u)(t)-\left(F_{\epsilon} u\right)(t)\right\| \leq\left\{\begin{array}{l}
M \int_{t-\epsilon}^{t}\left(a(s)+L_{f}(s) R^{\gamma}+\int_{0}^{s} q(s-\tau)\left(b(\tau)+L_{h}(\tau) R^{\mu}\right) d \tau\right) d s, \\
\quad t \in\left[0, t_{1}\right], \\
0, \quad t \in\left(t_{i}, s_{i}\right], i=1,2, \ldots, m, \\
M \int_{t-\epsilon}^{t}\left(a(s)+L_{f}(s) R^{\gamma}+\int_{0}^{s} q(s-\tau)\left(b(\tau)+L_{h}(\tau) R^{\mu}\right) d \tau\right) d s, \\
t \in\left(s_{i}, t_{i+1}\right], i=1, \ldots, m,
\end{array}\right.
$$

we get $Y(t)=\left\{(F u)(t): u \in B_{R}\right\}$ is totally bounded. Thus, $Y(t)$ is relatively compact in $E$. By using the Arzelá-Ascoli theorem, we have that $F: \operatorname{PC}(J, E) \rightarrow \operatorname{PC}(J, E)$ is completely continuous.

For $0<\lambda<1$, let $u=\lambda(F u)$, we have

$$
u(t)=\left\{\begin{array}{l}
\lambda U_{\beta}(t, 0)\left[U_{\beta}\left(T, t_{m}\right) g_{m}\left(s_{m}, u\left(s_{m}\right)\right)\right. \\
\left.\quad+\lambda \int_{s_{m}}^{T} U_{\beta}(T, s)\left(f(s, u(s))+\int_{0}^{s} q(s-\tau) h(\tau, u(\tau)) d \tau\right) d s\right] \\
\quad+\lambda \int_{0}^{t} U_{\beta}(t, s)\left(f(s, u(s))+\int_{0}^{s} q(s-\tau) h(\tau, u(\tau)) d \tau\right) d s, \quad t \in\left[0, t_{1}\right], \\
\lambda U_{\beta}\left(t, t_{i}\right) g_{i}(t, u(t)), \quad t \in\left(t_{i}, s_{i}\right], i=1,2, \ldots, m, \\
\lambda U_{\beta}\left(t, t_{i}\right) g_{i}\left(s_{i}, u\left(s_{i}\right)\right)+\lambda \int_{s_{i}}^{t} U_{\beta}(t, s)\left(f(s, u(s))+\int_{0}^{s} q(s-\tau) h(\tau, u(\tau)) d \tau\right) d s, \\
\quad t \in\left(s_{i}, t_{i+1}\right], i=1, \ldots, m,
\end{array}\right.
$$

and then

$$
\|u(t)\| \leq\left\{\begin{array}{l}
M^{2}\left(\bar{C}+\bar{L} R^{v_{m}}\right)+M^{2} \int_{s_{m}}^{T}\left(a(s)+L_{f}(s) R^{\gamma}+\int_{0}^{s} q(s-\tau)\left(b(\tau)+L_{h}(\tau) R^{\mu}\right) d \tau\right) d s \\
\quad+M \int_{0}^{t}\left(a(s)+L_{f}(s) R^{\gamma}+\int_{0}^{s} q(s-\tau)\left(b(\tau)+L_{h}(\tau) R^{\mu}\right) d \tau\right) d s, \quad t \in\left[0, t_{1}\right] \\
M\left(\bar{C}+\bar{L} R^{v_{i}}\right), \quad t \in\left(t_{i}, s_{i}\right], i=1,2, \ldots, m \\
M\left(\bar{C}+\bar{L} R^{v_{i}}\right)+M \int_{s_{i}}^{t}\left(a(s)+L_{f}(s) R^{\gamma}+\int_{0}^{s} q(s-\tau)\left(b(\tau)+L_{h}(\tau) R^{\mu}\right) d \tau\right) d s \\
\quad t \in\left(s_{i}, t_{i+1}\right], i=1, \ldots, m .
\end{array}\right.
$$

Let

$$
\begin{aligned}
\rho= & \max \left\{M^{2}\left(\bar{C}+\bar{L} R^{v_{m}}\right)\right. \\
& +M^{2} \int_{s_{m}}^{T}\left(a(s)+L_{f}(s) R^{\gamma}+\int_{0}^{s} q(s-\tau)\left(b(\tau)+L_{h}(\tau) R^{\mu}\right) d \tau\right) d s \\
& +M \int_{0}^{t}\left(a(s)+L_{f}(s) R^{\gamma}+\int_{0}^{s} q(s-\tau)\left(b(\tau)+L_{h}(\tau) R^{\mu}\right) d \tau\right) d s, \\
& M\left(\bar{C}+\bar{L} R^{v_{i}}\right), \\
& M\left(\bar{C}+\bar{L} R^{v_{i}}\right) \\
& +M \int_{s_{i}}^{t}\left(a(s)+L_{f}(s) R^{\gamma}+\int_{0}^{s} q(s-\tau)\left(b(\tau)+L_{h}(\tau) R^{\mu}\right) d \tau\right) d s, \\
& \left.t \in\left(s_{i}, t_{i+1}\right], i=1, \ldots, m\right\} .
\end{aligned}
$$


Then there exists a constant $M_{*}>\rho$ such that $\|u\|_{\mathrm{PC}} \neq M_{*}$. Let $V=\left\{u \in \mathrm{PC}(J, E):\|u\|_{\mathrm{PC}}<\right.$ $\left.M_{*}\right\}$. Obviously there is no $u \in \partial V$ such that $u=\lambda(F u)$ for $\lambda \in(0,1)$. It thus follows from the nonlinear alternative for single-valued maps that $F$ has a fixed point $u$ in $\bar{V}$, which is a mild solution of problem (1.1).

\section{An example}

We give an example to illustrate our main results in this paper.

$$
\left\{\begin{array}{l}
{ }^{c} D_{t}^{\alpha} u(x, t)=t \frac{\partial^{2}}{\partial x^{2}} u(x, t)+\frac{t}{1+t^{2}} \sin u(x, t)+\int_{0}^{t} e^{t-s} \frac{e^{s}}{\|u(x, s)\|} d s, \\
\quad t \in[0,1) \cup(2,3], \\
\frac{\partial}{\partial x} u(0, t)=\frac{\partial}{\partial x} u(1, t)=0, \quad t \in[0,1) \cup(2,3], \\
u(x, t)=U_{\beta}(t, 1) \frac{1}{3} u(x, t), \quad x \in[0, \pi], t \in(1,2], x \in(0,1), \\
u(0, x)=u(3, x)=0, \quad x \in(0,1),
\end{array}\right.
$$

where $E=L^{2}[0,3], J=[0,3], 0=t_{0}=s_{0}, t_{1}=1, s_{1}=2, T=3,0<\alpha \leq 1$, the operator $A: D(A)=\left\{u \in E: u^{\prime \prime} \in E, u(0)=u(1)=0\right\} \subset E \rightarrow E$ defined by $A(t)(z)=t \frac{\partial^{2} u}{\partial x^{2}}$. Then $A(t)$ generates a $\beta$-resolvent family $U_{\beta}(t, s)$ on $E$.

Denote

$$
\begin{aligned}
& u(t)=u(\cdot, t), \quad f(t, u(t))=\frac{t}{1+t^{2}} \sin u(\cdot, t), \quad h(t, u(t))=\frac{e^{s}}{\|u(\cdot, t)\|}, \\
& q(t-s)=e^{t-s}, \quad g_{1}(t, u(t))=\frac{1}{3} u(\cdot, t) .
\end{aligned}
$$

Therefore, Eq. (5.1) takes the following abstract form:

$$
\left\{\begin{array}{l}
{ }^{c} D_{t}^{\beta} u(t)=A(t) u(t)+f(t, u(t))+\int_{0}^{t} q(t-s) h(s, u(s)) d s, \\
\quad t \in\left(s_{i}, t_{i+1}\right], i=0,1, \ldots, m, \\
u(t)=U_{\beta}\left(t, t_{i}\right) g_{i}(t, u(t)), \quad t \in\left(t_{i}, s_{i}\right], i=1,2, \ldots, m, \\
u(0)=u(T) .
\end{array}\right.
$$

If we assume that problem (5.2) satisfies the conditions of Theorems 3.1 and 4.1, then by Theorems 3.1 and 4.1, we know that problem (5.2) has a mild solution, which means that problem (5.1) has a mild solution.

\section{Conclusion}

This paper investigates the existence of periodic boundary value problems for fractional semilinear integro-differential equations with non-instantaneous impulses by the measure of noncompactness, the theory of the resolvent family, and the fixed point theorem. The main results presented in this paper improve and generalize many results in [18-20, 24-26].

\section{Acknowledgements}

The authors would like to thank the referee for very important comments, which improved the results and the quality of the paper. 


\section{Funding}

This work was supported by the Project of Shandong Province Higher Educational Science and Technology Program (No. J16LI14), and by the National Natural Science Foundation of China (No. 11871302).

\section{Availability of data and materials}

Data sharing not applicable to this article as no datasets were generated or analysed during the current study.

\section{Ethics approval and consent to participate}

Not applicable.

\section{Competing interests}

The authors declare that they have no competing interests.

\section{Authors' contributions}

All authors contributed equally and significantly in writing this article. All authors read and approved the final manuscript.

\section{Author details}

'School of Mathematic and Quantitative Economics, Shandong University of Finance and Economics, Jinan, P.R. China.

${ }^{2}$ School of Mathematical Sciences, Qufu Normal University, Qufu, P.R. China. ${ }^{3}$ Department of Mathematics and Statistics, Curtin University, Perth, Australia.

\section{Publisher's Note}

Springer Nature remains neutral with regard to jurisdictional claims in published maps and institutional affiliations.

\section{Received: 10 January 2018 Accepted: 6 August 2018 Published online: 17 August 2018}

\section{References}

1. Afonso, S., Bonotto, M., Federson, M., Gimenes, P.: Stability of functional differential equations with variable impulsive perturbations via generalized ordinary differential equations. Bull. Sci. Math. 137, 189-214 (2013)

2. Afonso, S., Bonotto, M., Federson, M., Schwabik, Š.: Discontinuous local semiflows for Kurzweil equations leading to LaSalle's invariance principle for differential systems with impulses at variable times. J. Differ. Equ. 250, 2969-3001 (2011)

3. Chadha, A., Pandey, D.: Existence results for an impulsive neutral stochastic fractional integro-differential equation with infinite delay. Nonlinear Anal. 128, 149-175 (2015)

4. Chauhan, A., Dabas, J.: Local and global existence of mild solution to an impulsive fractional functional integro-differential equation with nonlocal condition. Commun. Nonlinear Sci. Numer. Simul. 19, 821-829 (2014)

5. Debbouche, A., Baleanu, D.: Controllability of fractional evolution nonlocal impulsive quasilinear delay integro-differential systems. Comput. Math. Appl. 62, 1442-1450 (2011)

6. Gou, H.D., Li, B.L.: Local and global existence of mild solution to impulsive fractional semilinear integro-differential equation with noncompact semigroup. Commun. Nonlinear Sci. Numer. Simul. 42, 204-214 (2017)

7. Ge, F.D., Zhou, H.C., Kou, C.H.: Approximate controllability of semilinear evolution equations of fractional order with nonlocal and impulsive conditions via an approximating technique. Appl. Math. Comput. 275, 107-120 (2016)

8. Shu, X.B., Lai, Y.Z., Chen, Y.M.: The existence of mild solutions for impulsive fractional partial differential equations. Nonlinear Anal. 74, 2003-2011 (2011)

9. Yan, Z.M., Lu, F.X.: Approximate controllability of a multi-valued fractional impulsive stochastic partial integro-differential equation with infinite delay. Appl. Math. Comput. 292, 425-447 (2017)

10. Zhu, B., Liu, L.S., Wu, Y.H.: Local and global existence of mild solutions for a class of nonlinear fractional reaction-diffusion equations with delay. Appl. Math. Lett. 61, 73-79 (2016)

11. Zhu, B., Liu, L.S., Wu, Y.H.: Local and global existence of mild solutions for a class of semilinear fractional integro-differential equations. Fract. Calc. Appl. Anal. 20(6), 1338-1355 (2017)

12. Cui, Y.J.: Uniqueness of solution for boundary value problems for fractional differential equations. Appl. Math. Lett. 51, 48-54 (2016)

13. Bai, Z.B., Zhang, S., Sun, S.J., Yin, C.: Monotone iterative method for fractional differential equations. Electron. J. Differ. Equ. 2016, 6 (2016)

14. Jiang, J.Q., Liu, L.S., Wu, Y.H.: Positive solutions for nonlinear fractional differential equations with boundary conditions involving Riemann-Stieltjes integrals. Abstr. Appl. Anal. 2012, Article ID 708192 (2012)

15. Bai, Z.B., Dong, X.Y., Yin, C.: Existence results for impulsive nonlinear fractional differential equation with mixed boundary conditions. Bound. Value Probl. 2016, 63 (2016)

16. Zhang, X.Q., Zhong, Q.: Uniqueness of solution for higher-order fractional differential equations with conjugate type integral conditions. Fract. Calc. Appl. Anal. 20(6), 1471-1484 (2017)

17. Zhang, X.Q., Zhong, Q.: Triple positive solutions for nonlocal fractional differential equations with singularities both on time and space variables. Appl. Math. Lett. 80,12-19 (2018)

18. Hernandez, E., O'Regan, D.: On a new class of abstract impulsive differential equations. Proc. Am. Math. Soc. 141, $1641-1649(2013)$

19. Pierri, M., O'Regan, D., Rolnik, V.: Existence of solutions for semilinear abstract differential equations with not instantaneous impulses. Appl. Math. Comput. 219, 6743-6749 (2013)

20. Yu, X., Wang, J.R.: Periodic boundary value for nonlinear impulsive evolution equations on Banach spaces. Commun. Nonlinear Sci. Numer. Simul. 22, 980-989 (2015)

21. Wang, J.R.: Stability of noninstantaneous impulsive evolution equations. Appl. Math. Lett. 73, 157-162 (2017)

22. Yang, D., Wang, J.R., O'Regan, D.: On the orbital Hausdorff dependence of differential equations with non-instantaneous impulses. C. R. Math. 356(2), 150-171 (2018) 
23. Wang, J.R., Fečkan, M., Tian, Y.: Stability analysis for a general class of non-instantaneous impulsive differential equations. Mediterr. J. Math. 14, 46 (2017)

24. Wang, J.R., Ibrahim, A.G., O'Regan, D.: Topological structure of the solution set for fractional non-instantaneous impulsive evolution inclusions. J. Fixed Point Theory Appl. 20, 1-25 (2018)

25. Anguraj, A., Kanjanadevi, S.: Non-instantaneous impulsive fractional neutral differential equations with state-dependent delay. Prog. Fract. Differ. Appl. 3, 207-218 (2017)

26. Kumar, P., Haloi, R., Bahuguna, D., Pandey, D.N.: Existence of solutions to a new class of abstract non-instantaneous impulsive fractional integro-differential equations. Nonlinear Dyn. Syst. Theory 16(1), 73-85 (2016)

27. Agarwal, R., Hristova, S., O'Regan, D.: Non-instantaneous Impulses in Differential Equations. Springer, Cham (2017)

28. Deimling, K.: Nonlinear Functional Analysis. Springer, New York (1985)

29. Caputo, M.: Linear models of dissipation whose $q$ is almost frequency independent. Geophys. J. R. Astron. Soc. 13(2), 529-539 (1967)

30. Kilbas, A., Srivastava, H., Trujillo, J.: Theory and Applications of Fractional Differential Equations. North-Holland Mathematics Studies, vol. 204. Elsevier, Amsterdam (2006)

31. Liu, L.S.: Iterative method for solutions and coupled quasi-solutions of nonlinear integro-differential equations of mixed type in Banach spaces. Nonlinear Anal. 42, 583-598 (2000)

32. Deimling, D.: Nonlinear Functional Analysis. Springer, Berlin (1985)

33. Liu, L.S., Guo, F., Wu, C.X.: Existence theorems of global solutions for nonlinear Volterra type integral equations in Banach spaces. J. Math. Anal. Appl. 309, 638-649 (2005)

34. Banas, J., Goebel, K.: Measure of Noncompactness in Banach Spaces. Lect. Notes Pure Appl. Math., vol. 60. Marcel Pekker, New York (1980)

35. Araya, D., Lizama, C.: Almost automorphic mild solutions to fractional differential equations. Nonlinear Anal. 69 3692-3705 (2008)

36. Pazy, A.: Semigroup of Linear Operators and Applications to Partial Differential Equations. Springer, New York (1983)

\section{Submit your manuscript to a SpringerOpen ${ }^{\circ}$ journal and benefit from:}

- Convenient online submission

- Rigorous peer review

- Open access: articles freely available online

- High visibility within the field

- Retaining the copyright to your article

Submit your next manuscript at $\boldsymbol{\nabla}$ springeropen.com 